\title{
Treatment of Community-Acquired Pneumonia: A Focus on Lefamulin
}

\author{
Nathaniel Eraikhuemen (D) - Daniel Julien · Alandra Kelly • \\ Taylor Lindsay $\cdot$ Dovena Lazaridis
}

Received: October 14, 2020 / Accepted: November 26, 2020 / Published online: February 2, 2021

(C) The Author(s) 2021

\begin{abstract}
Objective: The goal of this article is to review the clinical pharmacology, pharmacokinetics, efficacy, and safety of lemafulin.

Data Sources: We performed a systematic literature review using the search terms of lefamulin and BC-3781 in the PubMed and EMBASE databases. We also cross-referenced the pertinent articles and searched ClinicalTrials.gov to identify ongoing and nonpublished studies.

Study Selection and Data Extraction: Published data from 2005 to 2019 evaluating the clinical pharmacology, efficacy, and safety studies of lefamulin were analyzed.

Data Synthesis: In phase 3 clinical trials, two multicenter, randomized double-blinded studies-Lefamulin Evaluation Against Pneumonia 1 and 2 (LEAP 1 and 2)—compared the efficacy and safety of lemafulin with moxifloxacin in patients diagnosed with community-acquired bacterial
\end{abstract}

\section{N. Eraikhuemen $(\bowtie) \cdot$ A. Kelly $\cdot$ T. Lindsay}

Florida A\&M University College of Pharmacy and Pharmaceutical Sciences, Institute of Public Health, Davie, FL, USA

e-mail: nathan.Eraikhuemen@famu.edu

D. Julien

Memorial Regional Hospital South, Hollywood, FL, USA

D. Lazaridis

Department of Pharmacy, Memorial Regional

Hospital, Hollywood, FL, USA pneumonia (CABP). Lemafulin given in doses of $600 \mathrm{mg}$ orally or $150 \mathrm{mg}$ intravenously were reported to have comparable efficacy to those of moxifloxacin with or without linezolid in patients with CABP. After the trial, the lefamulin group had an early clinical response (ECR) of $87.3 \%$ and the moxifloxacin group had an ECR of $90.2 \%$. The difference of $-2.9 \%$ in the ECR was non-significant (CI - 8.5, 2.8).

Relevance to patients and clinical practice: Lemafulin exhibits a unique binding property; therefore, it possess a potentially lower predisposition for the development of bacterial resistance and cross-resistance to other antimicrobial classes. Lefamulin is active against gram-positive including methicillin-resistant strains and atypical organisms which are often implicated in CABP.Lefamulin may be a safe alternative for adult patients with CABP who may not be candidates for respiratory fluoroquinolones.Lefamulin demonstrates both bactericidal and bacteriostatic activity against gram-positive, fastidious gram-negatives, atypical pathogens, and some gram-negative anaerobes. It is bactericidal in vitro against Streptococcus pneumoniae, Haemophilus influenzae, and Mycoplasma pneumoniae (including macrolide-resistant strains) at concentrations of $0.06,0.5$, and $0.008 \mu \mathrm{g} / \mathrm{ml}$ respectively, and bacteriostatic against Staphylococcus aureus and Streptococcus pyogenes. The agent also demonstrates both time- and concentration-dependent killing against the pathogens $S$. pneumoniae and 
S. aureus.In vitro susceptibility testing demonstrated an $\mathrm{MIC}_{50 / 90}$ of $0.06 / 0.12 \mu \mathrm{g} / \mathrm{ml}$ against $S$. pneumoniae and S.aureus. The SENTRY Antimicrobial Surveillance Program found that at a concentration $\leq 1 \mu \mathrm{g} / \mathrm{ml}$, lefamulin inhibited $100 \%$ S. pneumoniae isolates, $99.8 \%$ of S. aureus isolates, and $99.6 \%$ of methicillin-resistant $S$. aureus isolates. It was not affected by resistance to various antibiotic classes such as beta-lactams, fluoroquinolones, or macrolides.

\section{PLAIN LANGUAGE SUMMARY}

Lefamulin is the first pleuromutilin antibiotic approved for the treatment of bacterial infections in humans. Pleuromutilin antibiotics exert their unique mechanism of action which makes them less susceptible to the development of bacterial resistance and low probability of cross-resistance to the other antimicrobial classes.

The authors present a critical review of the pharmacology, pharmacokinetics (PK), pharmacodynamics (PD), and data from two pivotal clinical trials of lefamulin in patients with community-acquired bacterial pneumonia (CABP).

Lefamulin exhibits both bactericidal and bacteriostatic activity against gram-positive, fastidious gram-negatives, atypical pathogens, and some gram-negative anaerobes. It has shown activity against organisms known to cause sexually transmitted infections, including Mycoplasma genitalium and drug-resistant Neisseria gonorrhoeae. Lefamulin demonstrated no activity against Enterobacteriaceae or Pseudomonas aeruginosa.

Pharmacokinetic studies involving lefamulin in acutely ill patients at least 18 years of age with three or more CABP symptoms failed to reveal any clinically significant differences in the PK parameters on the basis of age, sex, race, weight, or renal impairment. Lefamulin $600 \mathrm{mg}$ tablets had a mean oral bioavailability of $25 \%$. Consumption of high-fat meals may slightly reduce the blood level of the drug.

In two phase 3 clinical trials, The Lefamulin Evaluation Against Pneumonia 1 and 2 (LEAP 1 and 2) compared the efficacy and safety of lemafulin with moxifloxacin in patients diagnosed with CABP. Lemafulin administered in doses of $600 \mathrm{mg}$ orally or $150 \mathrm{mg}$ intravenously were reported to have comparable efficacy to those of moxifloxacin with or without linezolid in patients with CABP.

Keywords: BC-3781; Community-Acquired pneumonia; Clinical pharmacology; Lemafulin; Mechanism of action

\section{Key Summary Points}

Lemafulin development emerged following the need to combat the limitations of the currently available drugs to treat community-acquired bacterial pneumonia (CABP).

Lemafulin's unique binding property makes it less likely to develop bacterial resistance and cross-resistance to other antimicrobial classes.

Pleuromutilin antibiotics exert their action by binding to the peptidyl transferase center of the 50S ribosome via several interactions, thereby preventing the binding of transfer ribonucleic acid (tRNA) for peptide transfer and inhibiting protein synthesis.

Lefamulin is active against gram-positive including methicillin-resistant strains and atypical organisms which are often implicated in CABP.

In two-phase 3 clinical trials, lemafulin given in doses of $600 \mathrm{mg}$ orally or $150 \mathrm{mg}$ intravenously produced comparable efficacy to those of moxifloxacin with or without linezolid in patients with CABP.

Lemafulin may be a reasonable option for patients with CABP who are intolerant to beta-lactams, fluoroquinolones, and macrolides. 


\section{DIGITAL FEATURES}

This article is published with digital features, including a summary slide and plain language summary, to facilitate understanding of the article. To view digital features for this article go to https://doi.org/10.6084/m9.figshare. 13286402 .

\section{INTRODUCTION}

Community-acquired pneumonia (CAP) is one of the leading causes of morbidity and mortality worldwide. In 2018, pneumonia was the second leading cause of hospitalization [1]. In the USA, CAP accounts for over 1.5 million hospitalizations and results in more than 14.2 million ambulatory care visits annually [1-3]. It is also the most common infectious cause of death in the USA, accounting for over 50,000 deaths annually $[2,4]$. The direct annual cost of CAP in the USA has been estimated to be at least US\$17 billion, with a mean cost per hospitalization of US $\$ 13,000[5,6]$. The average length of stay for the treatment of pneumonia is 5.2 days [4]. This contributes to days of work missed and loss of productivity for both patients and caregivers.

CAP is an acute infection of the lung parenchyma characterized by dyspnea, cough, sputum production, chest pain, symptoms of infection such as fever or malaise, and the presence of patchy opacities upon radiographic visualization [7]. Risk factors for pneumonia include age, lifestyle factors, and certain comorbidities. Persons 2 years of age or younger and those over 65 years of age are at the highest risk for succumbing to the disease [8]. Lifestyle factors such as smoking, excessive alcohol intake, and having regular contact with children also increase the risk of developing CAP. Comorbid conditions such as chronic obstructive pulmonary disease and immunocompromising conditions such as the human immunodeficiency virus/acquired immunodeficiency syndrome (HIV/AIDS) can increase the risk of developing pneumonia [9].

Community-acquired bacterial pneumonia (CABP) is one of the most common forms of pneumonia. Infection can be caused by grampositive, gram-negative, or atypical organisms.

Hospital-acquired pneumonia (HAP), also referred to as nosocomial pneumonia, is pneumonia acquired during or after hospitalization for another illness or procedure with the onset of at least $48 \mathrm{~h}$ after hospital admission. The causative pathogens, treatment, and the prognosis are different from those of CAP. Ventilator-associated pneumonia (VAP) is a subclass of HAP. VAP is pneumonia which occurs at least 48-72 $\mathrm{h}$ after intubation and mechanical ventilation [10]. The exact incidence of HAP varies widely depending on the case definition of pneumonia and the population being studied. HAP accounts for up to $25 \%$ of all intensive care unit (ICU) infections and VAP occurs in $9-27 \%$ of all intubated patients [10].

Typical causative bacterial organisms of CAP include Streptococcus pneumoniae, Mycoplasma pneumoniae, Haemophilus influenzae, Staphylococcus aureus, Moraxella catarrhalis, Chlamydia pneumoniae, and Legionella pneumophila; $S$. pneumoniae is the most common causative bacteria pathogen $[11,12]$.

Pharmacological treatment of pneumonia is dependent on the patient's comorbidities, risk factors, the severity of illness, and the local resistance patterns. As per the 2019 American Thoracic Society, standard pharmacological treatment of non severe inpatient pneumonia includes a $\beta$-lactam in combination with macrolide or monotherapy with a respiratory fluoroquinolone [13].

Although there are various agents available for the treatment of CAP, there are limitations to many of them. Current treatment limitations include allergies, antibiotic resistance, inadequate penetration in lung tissues, and undesirable adverse effects, such as Clostridioides difficile (C. diff) diarrhea [14]. $\beta$-Lactam allergies are some of the most common self-reported allergies with a reported incidence of $17 \%$ in hospitalized patients. Macrolide and fluoroquinolone allergies are less common; however, they do occur [15-17].

The pleuromutilin antibiotics have a unique structure and mechanism of action, which results in no cross-sensitivity between other classes; this makes them viable options for 
patients who are allergic to the other agents. A macrolide resistance rate of more than $30 \%$ was found amongst S. pneumoniae isolates [18]. Macrolide resistance occurs via two main mechanisms, the first being a mutation that codes for antibiotic efflux pumps that remove the antibiotic from the target sites. The second resistance mechanism results from alterations of the bacterial ribosome, resulting in a phenotype with reduced susceptibility to macrolides, lincosamides, and streptogramin B. The prevalence of each resistance mechanism varies on the basis of geographic region. Lefamulin has activity against macrolide-resistant strains [19]. Another shortcoming of the current medications is the lack of adequate lung penetration. For instance, agents such as vancomycin, daptomycin and aminoglycosides exhibit poor penetration into the epithelial lining fluid, thus resulting in treatment failure $[20,21]$. Lefamulin is unaffected by pulmonary surfactants and was found to reach adequate levels in epithelial lining fluid [22]. Undesirable side effects common with standard pneumonia therapies include cardiac arrhythmias, which are associated with macrolides [13] and fluoroquinolones. Other side effects such as hypoglycemia, QTc prolongation, aortic dissection, and psychiatric side effects have all been reported with fluoroquinolones [23-25].

To combat the limitations of the available pharmacotherapeutic agents, novel therapies for the treatment of CABP are warranted. In August 2019, the US Food and Drug Administration (FDA) approved lefamulin for oral and intravenous administration for the treatment of adults with CABP caused by susceptible bacterial organisms [26].

\section{Data Sources}

We performed a systematic literature review using the following search terms: lefamulin, BC378, pharmacology, pharmacokinetics, and clinical studies in the PubMed and EMBASE databases. Also, we cross-referenced the pertinent articles and searched ClinicalTrials.gov to identify ongoing studies.

\section{Study Selection and Data Extraction}

Published articles in the English language from 2005 to 2019 evaluating the clinical pharmacology, pharmacokinetics efficacy, and safety studies of lefamulin were assessed and evaluated.

\section{CLINICAL PHARMACOLOGY}

Pleuromutilin antibiotics are derived from basidiomycete fungi. Lefamulin is the first pleuromutilin to be approved for systemic use in humans. The first pleuromutilin marked in 1979 was tiamulin, but was intended for veterinary use only [27]. Pleuromutilin antimicrobial agents such as tiamulin and valnemulin are used mostly in swine and to a lesser extent in poultry and rabbits. These agents are indicated for swine dysentery, spirochete-associated diarrhea, porcine proliferative enteropathy, enzootic pneumonia, and other infections where Mycoplasma organisms are involved [28].

In 2007, retapamulin was made available for topical use in humans [29].

Lefamulin is a semisynthetic antibiotic indicated for the treatment of adults with CABP caused by $S$. pneumoniae, methicillin-susceptible isolates of S.aureus, H. influenzae, L.pneumophila, M.pneumoniae, and Chlamydophila pneumoniae [11].

\section{Mechanism of Action}

Lefamulin is the first pleuromutilin antibiotic approved for the systemic treatment of bacterial infections in humans. Pleuromutilin antibiotics exert their action by binding to the peptidyl transferase center of the 50S ribosome via several interactions, including four hydrogen bonds. A binding pocket is formed and the bacterial ribosome closes around the mutulin core, preventing the binding of transfer ribonucleic acid (tRNA) for peptide transfer and therefore inhibiting protein synthesis $[13,30]$. Owing to its unique binding sites, there is a lower predisposition for the development of bacterial resistance. This unique mechanism of 
action also allows for a low probability of crossresistance to the other antimicrobial classes.

Lefamulin exhibits both bactericidal and bacteriostatic activity against gram-positive, fastidious gram-negatives, atypical pathogens, and some gram-negative anaerobes. It has shown activity against organisms known to cause sexually transmitted infections, including Mycoplasma genitalium and drug-resistant Neisseria gonorrhoeae. Lefamulin demonstrated no activity against Enterobacteriaceae or Pseudomonas aeruginosa [31].

\section{Pharmacokinetics}

Pharmacokinetic studies involving lefamulin in acutely ill patients at least 18 years of age with three or more CABP symptoms revealed no clinically significant differences in the pharmacokinetic parameters on the basis of age, sex, race, weight, or renal impairment $[19,32]$.

\section{Absorption}

Lefamulin $600 \mathrm{mg}$ tablets had a mean oral bioavailability of $25 \%$. Peak plasma concentration following oral administration was reached between 0.88 and $2 \mathrm{~h}$ in healthy subjects. Consumption of high-fat meals consisting of at least $50 \%$ fat or high-calorie meals (800-$1000 \mathrm{kcal})$ resulted in slightly reduced bioavailability $[19,33]$. The absolute oral bioavailability is $25.8 \%$ in the fasted state and $21.0 \%$ in the fed state[34]. The reported free plasma area under the concentration-time curve over $24 \mathrm{~h}$ $\left(f \mathrm{AUC}_{0-24}\right)$ and the free plasma maximum concentration $\left(C_{\max }\right)$ were $1500.8 \mathrm{mg} \mathrm{h} / \mathrm{L}$ and $330.1 \mathrm{mg} / \mathrm{L}$. respectively.

\section{Distribution}

Lefamulin is highly protein-bound, between $94.8 \%$ and $97.1 \%[19,35]$. The mean volume of distribution was $86.1 \mathrm{~L}$ in patients following intravenous (IV) administration. After a single $150 \mathrm{mg}$ IV administration infused over $1 \mathrm{~h}$, the highest lefamulin epithelial lining fluid (ELF) concentrations were observed at the end of the infusion. The mean ELF concentration was 3.87 $\mu \mathrm{g} \mathrm{h} / \mathrm{ml}[19]$.

\section{Metabolism}

In vitro studies revealed that lefamulin is both a substrate and an inhibitor of the CYP3A4 isoenzyme $[19,33]$. Therefore, the administration of lefamulin should be avoided or administered with caution in patients taking medications that are metabolized by the CYP3A isoenzyme. A reduction in the IV dosage of lefamulin to $150 \mathrm{mg}$ every $24 \mathrm{~h}$ in patients with severe hepatic impairment (Child-Pugh class C) is strongly suggested. Lefamulin tablets have not been studied in patients with moderate to severe hepatic impairment, and therefore are not recommended [19].

\section{Elimination}

The mean total body clearance of lefamulin is $11.9 \mathrm{~L} / \mathrm{h}$ in patients with CABP after IV infusion $[19,33]$. The mean elimination half-life is approximately $8 \mathrm{~h}$ in patients with CABP $[11,36]$. Following IV administration of $150 \mathrm{mg}$ of lefamulin, $77 \%$ of the dose was excreted in the feces with approximately $4.2-9.1 \%$ unchanged and approximately $14.1-15.5 \%$ was excreted unchanged in the urine. Following oral administration of $600 \mathrm{mg}, 89 \%$ was excreted in the feces and 5\% was excreted in the urine [19].

\section{Pharmacodynamics}

Lefamulin exhibits primarily area under concentration to minimum inhibitory concentration (AUC/MIC) pharmacodynamic activity. Lefamulin is bactericidal in vitro against $S$. pneumoniae, $H$. influenzae, and M. pneumoniae (including macrolide-resistant strains) at concentrations of $0.06,0.5$, and $0.008 \mu \mathrm{g} / \mathrm{ml}$, respectively, and bacteriostatic against $S$. aureus and $S$. pyogenes [19] (Table 1).

Lefamulin demonstrates both time- and concentration-dependent killing against the pathogens S.pneumoniae and S.aureus [29]. In vitro susceptibility testing demonstrated an $\mathrm{MIC}_{50 / 90}$ of $\quad 0.06 / 0.12 \mu \mathrm{g} / \mathrm{ml}$ against 
S. pneumoniae and S. aureus [37]. The SENTRY Antimicrobial Surveillance Program found that at a concentration $1 \mu \mathrm{g} / \mathrm{ml}$ or lower, lefamulin inhibited $100 \%$ S. pneumoniae isolates, $99.8 \%$ of S. aureus isolates, and $99.6 \%$ of methicillin-resistant $S$. aureus isolates. It was not affected by resistance to various antibiotic classes such as beta-lactams, fluoroquinolones, or macrolides [38].

The requirement for bactericidal activity against mycoplasmal infections is a minimum bactericidal concentration (MBC) at most two dilutions greater than the minimum inhibitory concentration (MIC) [39]. Lefamulin had an MIC of at most $0.008 \mu \mathrm{g} / \mathrm{ml}$ against 18 macrolide-susceptible and 42 macrolide-resistant strains of M.pneumoniae. The MICs which inhibited $90 \%\left(\mathrm{MIC}_{90}\right.$ ) of macrolide-susceptible and macrolide-resistant M. pneumoniae were at most $0.001 \mu \mathrm{g} / \mathrm{ml}$ and $0.002 \mu \mathrm{g} / \mathrm{ml}$, respectively [40]. Lefamulin underwent susceptibility testing against other atypical pathogens such as L. pneumophila and C. pneumoniae [41]. Against L. pneumophila and C. pneumoniae lefamulin had an $\mathrm{MIC}_{50 / 90}$ of $0.06 / 0.5 \mu \mathrm{g} / \mathrm{ml}$ and $0.02 / 0.04 \mu \mathrm{g} /$ $\mathrm{ml}$, respectively.
The SENTRY Antimicrobial Surveillance Program recorded the in vitro activity of lefamulin against organisms such as H. influenzae and M. catarrhalis between 2015 and 2016 [42]. Against $H$. influenzae, lefamulin has an $\mathrm{MIC}_{50 / 90}$ of $0.5 / 1 \mu \mathrm{g} / \mathrm{ml}$ which was comparable to Augmentin $(0.5 / 2 \mu \mathrm{g} / \mathrm{ml})$. Against M. catarrhalis, lefamulin has an $\mathrm{MIC}_{50 / 90}$ of $0.06 / 0.12 \mu \mathrm{g} / \mathrm{ml}$ which was comparable to moxifloxacin (0.06/ $0.06 \mu \mathrm{g} / \mathrm{ml}$ ) [42]. In general, lefamulin demonstrates susceptibility against commonly encountered bacterial pathogens (Table 2).

\section{CRUCIAL CLINICAL STUDIES}

Lefamulin Evaluation Against Pneumonia 1 (LEAP 1) and Lefamulin Evaluation Against Pneumonia 2 (LEAP 2) phase 3 trials were pivotal in the assessment of lefamulin in patients with CABP.

Table 1 In vitro activities of lefamulin activity against common pathogens [18]

\begin{tabular}{|c|c|c|c|}
\hline & Gram-positive bacteria & $\begin{array}{l}\text { Gram-negative } \\
\text { bacteria }\end{array}$ & Other bacteria \\
\hline Activity shown in vitro and in clinical infections & $\begin{array}{l}\text { Streptococcus pneumoniae } \\
\text { Streptococcus aureus } \\
\text { (methicillin-susceptible } \\
\text { isolates) }\end{array}$ & $\begin{array}{c}\text { Haemophilus } \\
\text { influenzae }\end{array}$ & $\begin{array}{l}\text { Mycoplasma } \\
\text { pneumoniae } \\
\text { Chlamydophila } \\
\text { pneumoniae } \\
\text { Legionella } \\
\text { pneumophila }\end{array}$ \\
\hline $\begin{array}{l}\text { Activity in vitro, but safety and efficacy in treating } \\
\text { clinical infections has not been established in clinical } \\
\text { trials }\end{array}$ & $\begin{array}{l}\text { Streptococcus aureus } \\
\text { (methicillin-resistant } \\
\text { [MRSA] isolates) } \\
\text { Streptococcus agalactiae } \\
\text { Streptococcus anginosus } \\
\text { Streptococcus mitis } \\
\text { Streptococcus pyogenes } \\
\text { Streptococcus salivarius }\end{array}$ & $\begin{array}{l}\text { Haemophilus } \\
\text { parainfluenzae } \\
\text { Moraxella } \\
\text { catarrhalis }\end{array}$ & \\
\hline
\end{tabular}




\section{Lefamulin Evaluation Against Pneumonia 1 (LEAP 1) [43]}

LEAP 1 was conducted as a multicenter, randomized, double-blind, double-dummy, activecontrolled, parallel-group study in which 551 participants with CABP were randomly assigned (1:1) to receive either lefamulin or moxifloxacin. Participants received lefamulin $150 \mathrm{mg}$ IV every $12 \mathrm{~h}$, with the option of switching to an oral study drug after six doses if the pre-specified improvement criteria were met $(n=276)$, or moxifloxacin $400 \mathrm{mg}$ IV every $24 \mathrm{~h}$ $(n=275)$. Participants receiving moxifloxacin also received linezolid $600 \mathrm{mg}$ IV every $12 \mathrm{~h}$ if MRSA was suspected at screening. Linezolid placebo was added to lefamulin in such cases. If MRSA was no longer suspected after a baseline culture, linezolid or the placebo was discontinued. The length of therapy for the participants ranged from 5 to 10 days.

Participants were required to meet the following inclusion criteria: adults greater than the age of 18, radiographic imaging that was indicative of pneumonia, Pneumonia Outcomes Research Team (PORT) risk class III or higher, an illness that initially presented within 7 days of enrollment, and three or more CABP symptoms (dyspnea, new or increased cough, purulent sputum production, chest pain). Study participants were excluded from the trial if they fell into any of the following categories: received a dose of antibiotics for the current illness within 3 days of randomization, have been hospitalized for at least 2 days within 90 days of the onset of symptoms, have confirmed or suspected CABP caused by P. aeruginosa or any member of the Enterobacteriaceae family, and a noninfectious cause of pulmonary infiltrates.

At baseline, of the 551 participants, $86.7 \%$ were white, $7.9 \%$ Asian, and $4.1 \%$ black; $43.5 \%$ of the participants were at least 65 years old, and $59.8 \%$ were male.

The FDA primary endpoint was an early clinical response (ECR) in the intention-to-treat population (ITT) at $96 \pm 24 \mathrm{~h}$ after the first study drug dose [14]. At the conclusion of the trial, the lefamulin group had an ECR of $87.3 \%$ $(241 / 276)$ and the moxifloxacin group had an ECR of $90.2 \%(248 / 275)$. The difference of
$-2.9 \%$ in the ECR was non-significant (CI - 8.5, 2.8). The European Medicines Agency (EMA) co-primary endpoint was an investigator assessment of clinical response (IACR) at test of cure (TOC) (5-10 days after last study drug dose) in the modified ITT (mITT) and clinically evaluable (CE) populations. Lefamulin demonstrated noninferiority for the EMA primary endpoint of IACR. The lefamulin treatment group had an IACR (mITT) of $80.8 \%$ versus $83.6 \%$ in the moxifloxacin treatment group. The difference of $-2.8 \%$ in the IACR (mITT) was non-significant (CI - 9.6, 3.9). Additionally, the lefamulin group had an IACR CE-TOC of $86.9 \%$ versus $89.4 \%$ in the moxifloxacin group. The difference of $-2.5 \%$ in the IACR CETOC group was insignificant (CI - 8.4, 3.4).

The differences in ECR in the treatment of class III and IV CABP were non-significant. As a result of the small sample size $(<10)$, the differences in ECR could not be evaluated in class II and V CABP. Lefamulin met the study criteria for noninferiority (Table 3 ).

\section{Lefamulin Evaluation Against Pneumonia 2 (LEAP 2) [44]}

LEAP 2 was a randomized, non-inferiority, double-blind, double-dummy, multicenter, parallel-group study comprising 738 participants. A total of 370 participants with confirmed CABP received $600 \mathrm{mg}$ PO every $12 \mathrm{~h}$ for 5 days in the lefamulin group and 368 participants received $400 \mathrm{mg}$ PO every $24 \mathrm{~h}$ for 7 days in the moxifloxacin group.

Inclusion criteria were similar to those specified in LEAP 1. Patients were required to meet the following inclusion criteria: adults greater than the age of 18 , radiographic imaging that was indicative of pneumonia, PORT risk class III or higher, illness that initially presented within 7 days of enrollment, and three or more CABP symptoms (dyspnea, new or increased cough, purulent sputum production, chest pain). Patients were excluded from the trial if they fall into any of the following categories: received a dose of antibiotics for the current illness within 3 days of randomization, hospitalized for two or more days within 90 days of the onset of 
Table 2 Summary of Lefamulin susceptibility against pathogens [38], [41-43]

\begin{tabular}{|c|c|c|c|c|c|c|c|c|}
\hline & \multicolumn{2}{|c|}{ Lefamulin } & \multicolumn{2}{|c|}{ Linezolid } & \multicolumn{2}{|c|}{ Vancomycin } & \multicolumn{2}{|c|}{ Daptomycin } \\
\hline & $\begin{array}{l}\mathrm{MIC}_{50} \\
\mu \mathrm{g} / \mathrm{ml}\end{array}$ & $\begin{array}{l}\mathrm{MIC}_{90} \\
\mu \mathrm{g} / \mathrm{ml}\end{array}$ & $\begin{array}{l}\mathrm{MIC}_{50} \\
\mu \mathrm{g} / \mathrm{ml}\end{array}$ & $\begin{array}{l}\mathrm{MIC}_{90} \\
\mu \mathrm{g} / \mathrm{ml}\end{array}$ & $\begin{array}{l}\mathrm{MIC}_{50} \\
\mu \mathrm{g} / \mathrm{ml}\end{array}$ & $\begin{array}{l}\mathrm{MIC}_{90} \\
\mu \mathrm{g} / \mathrm{ml}\end{array}$ & $\begin{array}{l}\mathrm{MIC}_{50} \\
\mu \mathrm{g} / \mathrm{ml}\end{array}$ & $\begin{array}{l}\mathrm{MIC}_{90} \\
\mu \mathrm{g} / \mathrm{ml}\end{array}$ \\
\hline \multicolumn{9}{|l|}{ Gram positive bacteria } \\
\hline $\begin{array}{l}\text { Staphylococcus aureus } \\
(n=5327)\end{array}$ & 0.12 & 0.12 & 1 & 1 & 1 & 1 & 0.25 & 0.5 \\
\hline MRSA & 0.12 & 0.25 & 1 & 1 & 1 & 1 & 0.25 & 0.5 \\
\hline $\begin{array}{l}\text { B-Hemolytic } \\
\text { streptococcus }(n=763)\end{array}$ & 0.03 & 0.03 & 1 & 1 & 0.25 & 0.5 & $\leq 0.06$ & 0.5 \\
\hline $\begin{array}{l}\text { Streptococcus Pyogen } \\
\text { group A }\end{array}$ & 0.03 & 0.03 & 1 & 1 & 0.25 & 0.5 & $\leq 0.06$ & $\leq 0.06$ \\
\hline $\begin{array}{l}\text { Streptococcus agalactiae, } \\
\text { group B }\end{array}$ & 0.03 & 0.03 & 1 & 1 & 0.5 & 0.5 & 0.12 & 0.25 \\
\hline $\begin{array}{l}\text { Streptococcus Viridans } \\
(n=245)\end{array}$ & 0.12 & 0.5 & 1 & 1 & 0.5 & 0.5 & 0.25 & 0.5 \\
\hline $\begin{array}{l}\text { Enterococcus faecium } \\
(n=536)\end{array}$ & 0.12 & 4 & 1 & 1 & $>16$ & $>16$ & 2 & 2 \\
\hline $\begin{array}{l}\text { Streptococcus pneumonia } \\
\quad(n=1473)\end{array}$ & 0.12 & 0.25 & 1 & 1 & 0.25 & 0.5 & - & - \\
\hline \multicolumn{9}{|l|}{ Gram negative bacteria } \\
\hline $\begin{array}{l}\text { Haemophilus influenzae } \\
(n=360)\end{array}$ & 1 & 2 & $\leq 0.05$ & $\leq 0.05$ & 1 & 2 & $\leq 0.06$ & $\leq 0.06$ \\
\hline $\begin{array}{l}\text { Moraxella catarrhalis } \\
(n=253)\end{array}$ & 0.12 & 0.25 & $\leq 0.05$ & $\leq 0.05$ & $\leq 0.25$ & $\leq 0.06$ & $\leq 0.25$ & $\leq 0.5$ \\
\hline \multicolumn{9}{|l|}{ Atypical organisms } \\
\hline $\begin{array}{l}\text { Legionella pneumophila } \\
(n=30)\end{array}$ & 0.12 & 0.5 & 0.06 & 0.12 & 0.06 & 0.12 & - & - \\
\hline $\begin{array}{l}\text { Chlamydophila } \\
\text { pneumoniae }(n=50)\end{array}$ & 0.02 & 0.04 & - & - & - & - & - & - \\
\hline $\begin{array}{l}\text { Mycoplasma pneumoniae } \\
(n=50)\end{array}$ & 0.006 & 0.006 & - & - & - & - & - & - \\
\hline
\end{tabular}

MRSA methicillin-resistant Staphylococcus aureus, $M I C$ minimum inhibitory concentrations

symptoms, at risk for major cardiac events or dysfunction, having significant hepatic disease, having confirmed or suspected CABP caused by MRSA, P. aeruginosa, or any member of the Enterobacteriaceae family, and a noninfectious cause of pulmonary infiltrates.
At baseline, of the 738 participants, $73.7 \%$ were white, 13.5\% Asian, 11.2\% Hispanic or Latino, and $5.5 \%$ black; $37.5 \%$ of the participants were at least 65 years old, 52.4\% were male, and 50.1\% were classified as having kidney impairment. Hypertension (36.2\%), asthma 
Table 3 Lefamulin Evaluation Against Pneumonia 1 (LEAP 1), $n=551$ [28]

Intervention

Lefamulin $150 \mathrm{mg} \mathrm{IVq12} \mathrm{h}(n=276)$

Early clinical response

$87.30 \%$

Response by pathogen

S. pneumoniae

S. aureus

H. influenzae

M. catarrhalis

M. pneumoniae

L. pneumophila

C. pneumoniae

Reported AE

$n=104$

$38.10 \%$

$2.90 \%$

$2.90 \%$

$2.90 \%$

$2.90 \%$

$2.20 \%$

$1.80 \%$

Most common AE

Hypokalemia

Nausea

Insomnia

Infusion site pain

Infusion site phlebitis
Moxifloxacin $400 \mathrm{mg}$ IV q24 h $(n=275)$

$90.20 \%$

S. pneumoniae

91/97 (93.8\%)

S. aureus

4/4 (100\%)

H. influenzae

$54 / 57$ (94.7\%)

M. catarrhalis

11/11 (100\%)

M. pneumoniae

$18 / 20(90.0 \%)$

L. pneumophila

$12 / 14(85.7 \%)$

C. pneumoniae

$18 / 19(94.7 \%)$

$n=103$

$37.70 \%$

Diarrhea

$7.70 \%$

Hypokalemia

$2.20 \%$

Nausea

$2.20 \%$

Rise in ALT

$2.20 \%$

Hypertension

$2.20 \%$

Insomnia

$1.80 \%$ or chronic obstructive pulmonary disease (16.7\%), and diabetes (13.4\%) comprised the most common preexisting conditions reported.

The FDA's primary endpoint was an ECR at $96 \mathrm{~h}( \pm 24 \mathrm{~h})$ after receipt of the first dose of either study drug in the ITT population. Lefamulin met the criteria for noninferiority to moxifloxacin. Both treatment groups had an ECR of 90.8 . The difference of $0.1 \%$ in the ECR was non-significant (CI $-4,4.5)$. As was the case in LEAP 1 trial, the EMA co-primary end points (FDA secondary end points) were IACR at TOC (5-10 days after last dose) in the mITT population and in the CE population. Rates of IACR success were found to be insignificant at $87.0 \%$ with lefamulin and $89.1 \%$ with moxifloxacin in the mITT population (difference $-2.1 \%$; CI $-7.0,2.8$ ) (Table 4 ).

\section{SAFETY CONSIDERATIONS}

\section{Adverse Events}

Adverse reactions such as injection site reactions, including infusion site pain, phlebitis, elevated transaminases, nausea, hypokalemia, and headache were reported by more than $2 \%$ of participants receiving lefamulin intravenously. In those receiving lefamulin orally, more than $2 \%$ of study participants experienced nausea, vomiting, diarrhea, and elevated 
Table 4 Lefamulin Evaluation Against Pneumonia 2 (LEAP 2) [39], $n=738$

Intervention

Lefamulin $600 \mathrm{mg}$ PO q12 h for 5 days $(n=370)$

Moxifloxacin $400 \mathrm{mg}$ PO q24 h for 7 days $(n=368)$

Early clinical response

$90.80 \%$

$90.80 \%$

Response by pathogen

S. pneumoniae

S. aureus

H. influenzae

M. catarrhalis

M. pneumoniae

L. pneumophila

C. pneumoniae

Reported AE

$n=120$

Most common AE

Diarrhea

Nausea

Vomiting

Hypertension

Viral respiratory tract infection
$12.20 \%$

$5.20 \%$

$3.30 \%$

$110 / 123(89.4 \%)$

$13 / 13(100 \%)$

$50 / 56(89.3 \%)$

$18 / 21(85.7 \%)$

$20 / 20(100 \%)$

$13 / 16(81.3 \%)$

$15 / 16(93.8 \%)$

$32.60 \%$

$1.40 \%$

$1.40 \%$
S. pneumoniae

S. aureus

H. influenzae

M. catarrhalis

M. pneumoniae

L. pneumophila

C. pneumoniae

$n=92$

Nausea

UTI

Headache

Hypertension
$1.90 \%$

$1.60 \%$

$1.60 \%$

$115 / 126(91.3 \%)$

$6 / 6(100 \%)$

$44 / 48(91.7 \%)$

$11 / 11(100 \%)$

$14 / 14(100 \%)$

$16 / 17(94.1 \%)$

$12 / 12(100 \%)$

$25.00 \%$

$1.40 \%$ transaminases. Less common adverse reactions include atrial fibrillation, anemia, thrombocytopenia, oropharyngeal and vulvovaginal candidiasis, anxiety, and urinary retention [19]. Lefamulin has the potential to prolong the QTc interval in some patients. C. difficile-associated diarrhea is a clinically significant adverse reaction that may occur following the oral or intravenous administration of lefamulin, as antibacterial agents alter the colon's normal flora and can cause an overgrowth of $C$. difficile [19]. In the LEAP 1 and LEAP 2 trials treatment discontinuation as a result of adverse events was $2.9 \%$ and $3.3 \%$ of participants, respectively $[43,44]$. The adverse events leading to treatment discontinuation included infusion site phlebitis, QTc prolongation, bradycardia, and severe vomiting.
The overall mean (standard deviation) change from baseline in QT interval corrected according to Fridericia (QTcF) on day 3 post dose was 13.8 (19.8) $\mathrm{ms}$ for lefamulin and 16.4 (21.4) $\mathrm{ms}$ for moxifloxacin. There were eight patients ( $n=3$ lefamulin; $n=5$ moxifloxacin) who presented with nonserious treatment emergent adverse events of prolonged QT intervals. However, in four patients ( $n=1$ lefamulin; $n=3$ moxifloxacin), the event led to study drug discontinuation [42].

\section{Drug-drug Interactions}

The concomitant oral or intravenous administration of lefamulin with strong and/or moderate CYP3A or P-glycoprotein inducers can 
reduce the efficacy of the drug and should be avoided. Conversely, oral or IV coadministration of lefamulin with drugs that strongly or moderately inhibit CYP3A or P-glycoprotein inhibitors may increase the risk of adverse reactions. Close monitoring of patients on concomitant therapy is warranted [19].

\section{Contraindications}

Lefamulin is contraindicated in patients with a known hypersensitivity to pleuromutilin drugs or to any of the agent's excipients. Lefamulin can prolong the QTc interval and should be avoided in patients with prolonged QTc. Lefamulin should be avoided in patients with a history of ventricular arrhythmias including torsades de pointes. Its use is therefore contraindicated with CYP3A4 inhibitors such as amiodarone, macrolides, verapamil, azoles, and protease inhibitors. Additionally, the coadministration of lefamulin with any of the following agents such as the class IA and class III antiarrhythmics, antipsychotics, tricyclic antidepressants, and the fluoroquinolones should be avoided [19].

\section{Use in specific populations}

The effects of lefamulin have not been studied in pregnant women. However, teratogenicity was demonstrated in animal studies involving lefamulin [19]. Therefore, pregnancy status should be assessed in women of childbearing age before starting lefamulin. Therapy with lefamulin may reduce the efficacy of oral contraceptives; therefore, women should use an additional forms of contraception during treatment and for at least 2 days after the completion of therapy. There are currently no studies evaluating the presence of lefamulin in human breast milk or its effect on a breastfed infant. To reduce the risk of serious adverse reactions, human milk should be discarded during treatment and for at least 2 days following treatment [19].

\section{DOSAGE AND ADMINISTRATION}

Lefamulin is indicated for the treatment of adults with CABP caused by the following susceptible microorganisms: S. pneumoniae, S. aureus (methicillin-susceptible isolates), $H$. influenzae, L. pneumophila, M. pneumoniae, and C. pneumoniae.

For the treatment of CABP, the recommended dose of lefamulin is $150 \mathrm{mg}$ infused intravenously over $60 \mathrm{~min}$ every $12 \mathrm{~h}$ for $5-$ 7 days. Alternatively, the drug can be administered orally with $600 \mathrm{mg}$ given every $12 \mathrm{~h}$ for 5 days. The oral tablets should be administered at least $1 \mathrm{~h}$ before or $2 \mathrm{~h}$ after meals and should be swallowed whole with $6-8 \mathrm{oz}$ of water. Lefamulin tablets should not be crushed or chewed. In patients with severe hepatic impairment (Child-Pugh class $\mathrm{C}$ ), the intravenous dose of lefamulin should be decreased to $150 \mathrm{mg}$ infused over $1 \mathrm{~h}$, every $24 \mathrm{~h}$. Lefamulin oral tablets have not been studied in those with moderate or severe hepatic impairment (Child-Pugh class B or C) and are not currently recommended in this population. Currently, there are no dosing adjustments for those with renal impairment or in patients who are on hemodialysis [19].

\section{P\&T Committee Considerations}

The American Thoracic Society CAP 2019 guidelines recommend amoxicillin, doxycycline, or a macrolide if resistance is below 25\% in patients without comorbidities or risk factors for MRSA/P. aeruginosa. Also, these patients should not have prior respiratory isolation of MRSA or P. aeruginosa or recent hospitalization and receipt of parenteral antibiotics in the last 90 days. The Society recommended treating patients with comorbidities such as chronic heart, lung, liver, or renal disease; diabetes mellitus; alcoholism; malignancy; or asplenia with a beta-lactam and a macrolide/doxycycline or monotherapy with a respiratory fluoroquinolone [13]. Lefamulin's place in therapy may be in patients who have contraindications or intolerances to the preferred regimens and where resistance to preferred treatment is high. 
Lefamulin is a single drug regimen with a novel mechanism of action. It is efficacious against S. pneumoniae, S. aureus (methicillin-susceptible isolates), H. influenzae, L. pneumophila, M. pneumoniae, and C. pneumoniae pathogens.

Renal and hepatic adjustment are not warranted; thus, lefamulin may be an alternative agent for patients with renal and hepatic insufficiency. Lefamulin is not associated with tendon rupture or neuropathy like the fluoroquinolones nor hepatotoxicity, nor dietary/drug-drug interactions with divalent cations like doxycycline and the fluoroquinolones.

Lefamulin should augment the current antimicrobial drugs to treat CABP. The high cost and prevalence of gastrointestinal side effects may make the widespread use of lefamulin in CABP implausible. However, there is a serious need for safe and effective oral options to treat bone, joint, and soft tissue infections particularly in outpatient settings.

\section{CONCLUSION}

Lefamulin is a novel pleuromutilin antibiotic with a broad spectrum of activity against grampositive and atypical organisms [14]. It is indicated for the treatment of adults with CABP caused by susceptible microorganisms.

The cost compared to the currently available agents for CABP may serve as a barrier to the use of lefamulin. The average wholesale price of the IV formulation of lefamulin is US\$205 per day, and the oral formulation will cost US $\$ 275$ per day, whereas older antibiotics are available at a fraction of this cost. Although lefamulin has been approved as an alternative therapy in the treatment of CAP, the American Thoracic Society and the Infectious Disease Society of America have recommended additional research in the outpatient setting $[13,26]$. Lefamulin is also undergoing several phases 1 trial for various indications, including prosthetic joint infections, sexually transmitted infections, osteomyelitis, and pediatric infections. In a phase 2 study, lefamulin was evaluated in 210 patients with acute bacterial skin and skin structure infection (ABSSSI) caused by a gram- positive pathogen. Randomized patients received either lefamulin $100 \mathrm{mg}$ or $150 \mathrm{mg}$, or vancomycin $1 \mathrm{~g}$ intravenously every $12 \mathrm{~h}$. Treatment response was assessed daily and at the TOC. Baseline characteristics, including the frequency of MRSA, were comparable between the different treatment groups. Clinical success at TOC in the clinically evaluative population occurred in $54(90.0 \%)$ patients in the lefamulin $100 \mathrm{mg}$ group, $48(88.9 \%)$ in the lefamulin $150-\mathrm{mg}$ group, and $47(92.2 \%)$ in the vancomycin group. On day 3 , the clinical response rate was similar across the three treatment groups. Lefamulin also demonstrated comparable clinical efficacy in ABSSSIs caused by grampositive pathogens, including MRSA, to those of vancomycin. The incidence rate for drug-related adverse events was lower for patients receiving lefamulin $(34.3 \%$ and $39.4 \%$ in the $100-\mathrm{mg}$ and 150 -mg groups, respectively) than those receiving vancomycin (53.0\%) [45]. The outcomes of these trials are forthcoming.

\section{ACKNOWLEDGEMENTS}

Funding. This review was supported by a grant from the National Institute of Minority Health and Health Disparity U54 MD 007582. The Rapid Service Fee was funded by the authors.

Authorship. All named authors meet the International Committee of Medical Journal Editors (ICMJE) criteria for authorship for this article, take responsibility for the integrity of the work as a whole, and have given their approval for this version to be published.

Disclosures. Nathaniel Eraikhuemen, Daniel Julien, Alandra Kelly, Taylor Lindsay and Dovena Lazaridis have nothing to disclose.

Compliance with Ethics Guidelines. This article is based on previously conducted studies and does not contain any new studies with human participants or animals performed by any of the authors. 
Open Access. This article is licensed under a Creative Commons Attribution-NonCommercial 4.0 International License, which permits any non-commercial use, sharing, adaptation, distribution and reproduction in any medium or format, as long as you give appropriate credit to the original author(s) and the source, provide a link to the Creative Commons licence, and indicate if changes were made. The images or other third party material in this article are included in the article's Creative Commons licence, unless indicated otherwise in a credit line to the material. If material is not included in the article's Creative Commons licence and your intended use is not permitted by statutory regulation or exceeds the permitted use, you will need to obtain permission directly from the copyright holder. To view a copy of this licence, visit http://creativecommons.org/licenses/by$\mathrm{nc} / 4.0 /$.

\section{REFERENCES}

1. American Thoracic Society-Patient Resources. https://www.thoracic.org/patients/patientresources/. Accessed Jan 31, 2020.

2. Sattar SBA, Sharma S. Bacterial pneumonia. StatPearls, Treasure Island. 2020. https://www.ncbi. nlm.nih.gov/books/NBK513321/. Accessed Dec 18 2019.

3. Agency for Healthcare Research and Quality. Community-acquired pneumonia in the emergency department setting. AHRQ. 2018. http://www.ahrq. gov/sites/default/files/wysiwyg/professionals/ quality-patient-safety/quality-resources/tools/captoolkit/cap_ed-pamphlet.pdf. Accessed June 15, 2020.

4. Ramirez J. Adults hospitalized with pneumonia in the United States: incidence epidemiology and mortality. Open Forum Infect Dis. 2017. https:// doi.org/10.1093/ofid/ofx163.1493.

5. File TM, Marrie TJ. Burden of community-acquired pneumonia in North American adults. Postgrad Med. 2010;122(2):130-41. https://doi.org/10.3810/ pgm.2010.03.2130.

6. Brown JD, Harnett J, Chambers R, Sato R. The relative burden of community-acquired pneumonia hospitalizations in older adults: a retrospective observational study in the United States. BMC
Geriatr. 2018;18(1):92. https://doi.org/10.1186/ s12877-018-0787-2.

7. Kolditz M, Ewig S. Community-acquired pneumonia in adults. Dtsch Arztebl Int. 2017;114(49): 838-48. https://doi.org/10.3238/arztebl.2017.0838.

8. Centers for Disease Control and Prevention. Pneumococcal disease | Transmission and those at high risk. CDC. https://www.cdc.gov/pneumococcal/ about/risk-transmission.html. Accessed Jan 28, 2020.

9. Torres A, Peetermans WE, Viegi G, Blasi F. Risk factors for community-acquired pneumonia in adults in Europe: a literature review. Thorax. 2013;68(11):1057-65. thoraxjnl-2013-204282.

10. American Thoracic Society, Infectious Diseases Society of America. Guidelines for the management of adults with hospital-acquired, ventilator-associated, and healthcare-associated pneumonia. Am J Respir Crit Care Med. 2005;17:388-416.

11. Regunath H, Oba Y. Community-acquired pneumonia. StatPearls, Treasure Island. 2019. https:// www.ncbi.nlm.nih.gov/books/NBK430749/. Accessed Jan 5, 2020.

12. Brown JS. Community-acquired pneumonia. Clin Med (Lond). 2012;12(6):538-43. https://doi.org/10. 7861/clinmedicine.12-6-538.

13. Metlay J, Waterer G, Long A, et al. Diagnosis and treatment of adults with community-acquired pneumonia. An official clinical practice guideline of the American Thoracic Society and Infectious Diseases Society of America. Am J Respir Crit Care Med. 2019;200(7):e45-67. https://doi.org/10.1164/rccm. 201908-1581st.

14. Ahdal J, Nayar S, Hasan A, Waghray P, Ramananthan S, Jain R. Management of community-acquired bacterial pneumonia in adults: limitations of current antibiotics and future therapies. Lung India. 2019;36(6):525. https://doi.org/10.4103/lungindia. lungindia_38_19.

15. Jeffres MN, Narayanan PP, Shuster JE, Schramm GE. Consequences of avoiding $\beta$-lactams in patients with $\beta$-lactam allergies. J Allergy Clin Immunol. 2016;137(4):1148-53. https://doi.org/10.1016/j. jaci.2015.10.026.

16. Hermanides J, Lemkes BA, Prins JM, Hollmann MW, Terreehorst I. Presumed $\beta$-lactam allergy and cross-reactivity in the operating theater. Anesthesiology. 2018;129(2):335-42. https://doi.org/10. 1097/aln.0000000000002252. 
17. Shaeer KM, Chahine EB, Varghese Gupta S, Cho JC. Macrolide allergic reactions. Pharmacy (Basel). 2019;7(3):135. pharmacy7030135.

18. Centers for Disease Control and Prevention. Active Bacterial Core Surveillance Report, Emerging Infections Program Network, Streptococcus pneumoniae, 2016. http://www.cdc.gov/abcs/reportsfindings/survreports/spneu16.pdf. Accessed Jun 22, 2020 .

19. XENLETA $^{\mathrm{TM}}$ (lefamulin) [package insert]. King of Prussia, PA: Nabriva Therapeutics; 2019.

20. Brun-Buisson C, Lemaire F. Administration of antibiotics for pneumonia during respiratory failure: reaching the target. Am J Respir Crit Care Med. 2001;164(9):1554-5. https://doi.org/10.1164/ ajrccm.164.9.2108099b.

21. Lodise TP, Drusano GL, Butterfield JM, Scoville J, Gotfried M, Rodvold KA. Penetration of vancomycin into epithelial lining fluid in healthy volunteers. Antimicrob Agents Chemother. 2011;55(12): 5507-11. https://doi.org/10.1128/aac.00712-11.

22. Wicha WW, Strickmann DB, Paukner S. Pharmacokinetics/pharmacodynamics of lefamulin in a neutropenic murine pneumonia model with Staphylococcus aureus and Streptococcus pneumoniae. J Antimicrobial Chemother. 2019;74(Supplement_ 3):iii11-iii18. https://doi.org/10.1093/jac/dkz086.

23. US Food and Drug Administration. FDA Drug Safety Communication: FDA reinforces safety information about serious low blood sugar levels and mental health side effects with fluoroquinolone antibiotics; requires label changes. FDA. 2018. https://www.fda. gov/drugs/drug-safety-and-availability. Accessed July 2, 2020

24. Center for Drug Evaluation and Research. Serious low blood sugar, new mental health effects with fluoroquinolones. US Food and Drug Administration. 2018. https://www.fda.gov/drugs/drug-safetyand-availability/fda-reinforces-safety-informationabout-serious-low-blood-sugar-levels-and-mentalhealth-side. Accessed July 2, 2020.

25. Center for Drug Evaluation and Research. FDA warns about increased risk of ruptures or tears in the aorta blood vessel with fluoroquinolone antibiotics in certain patients. US Food and Drug Administration. 2018. https://www.fda.gov/drugs/drug-safety-andavailability/fda-warns-about-increased-risk-rupturesor-tears-aorta-blood-vessel-fluoroquinolone-antibio tics. Accessed June 27, 2020.

26. Nabriva Therapeutics. Nabriva Therapeutics receives U.S. FDA approval of Xenleta ${ }^{\mathrm{TM}}$ (lefamulin) to treat community-acquired bacterial pneumonia
(CABP). 2019. Available at: https://investors. nabriva.com/news-releases/news-release-details/ nabriva-therapeutics-receives-us-fda-approvalxenleta. Accessed July 03, 2020.

27. Rodvold KA. Introduction: lefamulin and pharmacokinetic/pharmacodynamic rationale to support the dose selection of lefamulin. J Antimicrob Chemother. 2019;74(Supplement_3):iii2-iii4. https:// doi.org/10.1093/jac/dkz084.

28. van Duijkeren E, Greko C, Pringle M, et al. Pleuromutilins: use in food-producing animals in the European Union, development of resistance and impact on human and animal health. J Antimicrob Chemother. 2014;69:2022-31.

29. Paukner S, Riedl R. Pleuromutilins: potent drugs for resistant bugs-mode of action and resistance. Cold Spring Harb Perspect Med. 2017;7(1):a027110. https://doi.org/10.1101/cshperspect.a027110.

30. Eyal Z, Matzov D, Krupkin M, et al. A novel pleuromutilin antibacterial compound, its binding mode and selectivity mechanism. Sci Rep. 2016;6(1):39004. https://doi.org/10.1038/ srep39004.

31. Veve MP, Wagner JL. Lefamulin: review of a promising novel pleuromutilin antibiotic. Pharmacotherapy. 2018;38(9):935-46. https://doi.org/10. 1002/phar.2166.

32. File TM Jr, Goldberg L, Das A, et al. Efficacy and safety of intravenous-to-oral lefamulin, a pleuromutilin antibiotic, for the treatment of communityacquired bacterial pneumonia: the phase III lefamulin evaluation against pneumonia (LEAP 1) trial. Clin Infect Dis. 2019;69(11):1856-67. https://doi. org/10.1093/cid/ciz090.

33. Wicha WW, Prince WT, Lell C, Heilmayer W, Gelone SP. Pharmacokinetics and tolerability of lefamulin following intravenous and oral dosing. J Antimicrob Chemother. 2019;74(Supplement_3): iii19-26. https://doi.org/10.1093/jac/dkz087.

34. Wicha W, Lell C, Seltzer E, Prince WT, Gelone S. Pharmacokinetics and safety of an oral, immediaterelease (IR) tablet formulation of lefamulin in fed and fasted healthy subjects. Poster presented at 27th European Congress of Clinical Microbiology and Infectious Diseases, Vienna; 2017.

35. Dubey A, Maggo S, Karan A, Singh N, Bhaskaran S. Lefamulin: novel pleuromutilin drug for community acquired bacterial pneumonia. Int J Basic Clin Pharmacol. 2019;8:2783. https://doi.org/10.18203/ 2319-2003.ijbcp20195297.

36. Wicha WW, Ivezic-Schoenfeld Z, Novak R. Preclinical efficacy of BC-3781 in thigh and bacteremia 
infections caused by staphylococci. 50th Interscience Conference on Antimicrobial Agents and Chemotherapy; Abstract F1-2109; 2010.

37. Bhavnani SM, Zhang L, Hammel JP, et al. Pharmacokinetic/pharmacodynamic target attainment analyses to support intravenous and oral lefamulin dose selection for the treatment of patients with community-acquired bacterial pneumonia. J Antimicrob Chemother. 2019;74:III35-41. https://doi. org/10.1093/jac/dkz089.

38. Paukner S, Gelone SP, Arends SJR, Flamm RK, Sader HS. Antibacterial activity of lefamulin against pathogens most commonly causing communityacquired bacterial pneumonia: SENTRY Antimicrobial Surveillance Program (2015-2016). Antimicrob Agents Chemother. 2019;63(4):e02161-e2218. https://doi.org/10.1128/AAC.02161-18.

39. Waites KB, Nolte FS. Laboratory diagnosis of mycoplasmal infections. Washington: ASM; 2001.

40. Waites KB, Crabb DM, Duffy LB, Jensen JS, Liu Y, Paukner S. In vitro activities of lefamulin and other antimicrobial agents against macrolide-susceptible and macrolide-resistant Mycoplasma pneumoniae from the United States, Europe, and China. Antimicrob Agents Chemother. 2017;61(2):e02008e2016. https://doi.org/10.1128/AAC.02008-16.

41. Sader HS, Paukner S, Ivezic-Schoenfeld Z, Biedenbach DJ, Schmitz FJ, Jones RN. Antimicrobial activity of the novel pleuromutilin antibiotic BC3781 against organisms responsible for communityacquired respiratory tract infections (CARTIs). J Antimicrob Chemother. 2012;67(5):1170-5. https:// doi.org/10.1093/jac/dks001.

42. Paukner S, Sader HS, Ivezic-Schoenfeld Z, Jones RN. Antimicrobial activity of the pleuromutilin antibiotic BC-3781 against bacterial pathogens isolated in the SENTRY antimicrobial surveillance program in 2010. Antimicrob Agents Chemother. 2013;57(9): 4489-4495. https://doi.org/10.1128/AAC.00358-13.

43. File Jr TM, Goldberg L, Das A, et al. Efficacy and safety of intravenous-to-oral lefamulin, a pleuromutilin antibiotic, for the treatment of communityacquired bacterial pneumonia: the phase III Lefamulin Evaluation Against Pneumonia (LEAP 1) trial. Clin Infect Dis. 2019;69(11):1856-1867. https://doi.org/10.1128/AAC.00358-13.

44. Alexander E, Goldberg L, Das AF, et al. Oral lefamulin vs moxifloxacin for early clinical response among adults with community-acquired bacterial pneumonia: the LEAP 2 randomized clinical trial. JAMA. 2019;322:1661-71. https://doi.org/10.1001/ jama.2019.15468.

45. Prince WT, Ivezic-Schoenfeld Z, Lell C, et al. Phase II clinical study of BC-3781, a pleuromutilin antibiotic, in treatment of patients with acute bacterial skin and skin structure infections. Antimicrobial Agents Chemother. 2013;57(5):2087-94. 